\title{
Renin-angiotensin system in the pathogenesis of COVID-19 and possible drug targets
}

\author{
Sujay Halkur Shankar ${ }^{1}$, Vishal Vishwakarma ${ }^{1}$, Arvind Kumar ${ }^{1}$, Rohini Sharma ${ }^{1}$, and \\ Naveet Wig ${ }^{1}$ \\ ${ }^{1}$ All India Institute of Medical Sciences
}

May 6, 2020

\begin{abstract}
COVID-19 is characterized by fever, cough, shortness of breath, myalgia, and headache. The disease also takes a more severe form with life-threatening manifestations of acute respiratory distress syndrome (ARDS), acute cardiac injury, acute kidney injury, disseminated intravascular coagulopathy, and cytokine storm. It has been elucidated that like its predecessor, the SARS $\mathrm{CoV}$, the SARS CoV-2 utilizes the ACE2 receptor to enter cells. This knowledge brought into speculation the effects of a dysregulated Renin-Angiotensin system (RAS) in the pathogenesis of COVID-19. It has been proposed that the effects of a dysregulated RAS would lead to an inflammatory cascade and contribute to the cytokine storm that is central to the disease. This paper looks at the RAS pathway and the hypothesizes the possibility of a positive RAS feedback loop in the pathogenesis of COVID-19. We also propose possible drug targets for the treatment of COVID-19.
\end{abstract}

\section{Introduction}

COVID-19, the largest pandemic of the modern world, has surpassed its predecessors SARS and MERS by miles. What started in Wuhan, China, has spread worldwide within four months. It has infected over three million people and has claimed over two hundred thousand lives. COVID-19 is characterized by fever, cough, shortness of breath, myalgia, and headache. The disease also takes a more severe form with life-threatening manifestations of acute respiratory distress syndrome (ARDS), acute cardiac injury, acute kidney injury, disseminated intravascular coagulopathy, and cytokine storm.

Risk factors that tend to make individuals susceptible to severe disease include older age and co-morbidities such as hypertension, diabetes, and pre-existing heart disease. ${ }^{1}$ Another aspect of the course that has been noted is the rapid deterioration of apparently stable individuals within a matter of hours. This observation might call into consideration a positive feedback loop in the pathogenesis of the disease.

It has been elucidated that like its predecessor, the SARS CoV, the SARS CoV-2 utilizes the ACE2 receptor to enter cells. ${ }^{2}$ This knowledge brought into speculation the effects of a dysregulated Renin-Angiotensin system (RAS) in the pathogenesis of COVID-19. It has been proposed that the effects of a dysregulated RAS would lead to an inflammatory cascade and contribute to the cytokine storm that is central to the disease. ${ }^{3}$ This paper looks at the RAS pathway and the possibility of a positive RAS feedback loop in the pathogenesis of COVID-19.

\section{Pathogenesis of SARS CoV-2}

The SARS CoV-2 virus enters the body through the respiratory epithelial cells. The envelope spike protein (S protein) engages with the ACE2 receptors on epithelial cells. ${ }^{2}$ This fusion can either be mediated by an early pathway (involving the serine protease TMPRSS2) or an endocytic late pathway (involving the cathepsin B/L pathway). ${ }^{4}$ Viral replication and release probably lead to a period of viremia. This is evidenced by viral 
RNA being detected in the stool and urine of humans. ${ }^{5}$ Macaque autopsy studies have shown viral particle staining in the lungs, lymph nodes, and ileum, suggesting interaction with these cells. ${ }^{6}$ Replication increases viral load and is known to downregulate the ACE2 receptor. ${ }^{7}$

Longer duration of viral shedding has been noticed in older monkeys than younger ones. ${ }^{6}$ This might indicate a delayed immune response in older individuals to viral invasion. A delayed response could lead to greater ACE2 downregulation making older individuals susceptible to reaching a critical or tipping point in the pathogenesis.

\section{Physiology of the RAS axis}

The RAS pathway is depicted in Figure 1. The RAS is a cascade of complex polypeptides that were originally described in the context of hypertension, cardiovascular, and renal disease. Research now shows that RAS plays a central role in immunomodulation. ${ }^{8}$ Renin is the rate-limiting enzyme of the RAS axis. It is secreted as a pro-enzyme, prorenin. Renin converts circulating angiotensinogen into angiotensin I. Angiotensin I is then broken down into Angiotensin II by angiotensin-converting enzyme (ACE). Angiotensin II is the primary effector molecule through its receptors - AT1 and AT2. Angiotensin II is degraded by angiotensin-converting enzyme 2 (ACE2) into Ang (1-7).

While the ACE-Angiotensin II pathway exerts a pro-inflammatory effect, ACE2-Ang(1-7) plays a role in immune tempering. Similarly, the effect of angiotensin II on the AT1 receptor is mainly pro-inflammatory while the action on the AT2 receptor is anti-inflammatory. ${ }^{9}$

Renin and prorenin exert a distinct proinflammatory effect through a separate receptor - the prorenin receptor (PRR). ${ }^{10}$ Apart from the effects of circulating RAS, tissue-specific RAS pathways in the heart, lung, kidney, and blood vessels mediate organ-specific inflammation; first described in the kidney. ${ }^{11}$

\section{RAS pathway in aging and COVID-19}

The RAS pathway undergoes major physiological changes during development and aging. In fish models, PRR has been found to play a role in embryogenesis, with PRR knockouts exhibiting developmental defects. In adults, however, PRR is known to increase levels of pro-inflammatory cytokines independent of the angiotensin II pathway. ${ }^{12}$

Fetal and neonatal mice kidneys have a higher expression of AT2 receptors than AT1 receptors. In adult mice, however, AT1 receptors are more widely distributed with AT2 having a selective and minor role. ${ }^{13}$ There is an upregulation of AT1 receptors with aging in non-human primates. ${ }^{14}$ Sheep studies have demonstrated that the ACE/ACE2 receptor expression ratio is significantly lower in male neonatal sheep than in adult sheep. ${ }^{15}$ It has also been shown that ACE2 levels may decrease in the lungs of aged rats. ${ }^{16}$

The low ACE2 levels in addition to the downregulation of ACE2 by SARS CoV-2 might be the tipping point in the pathogenesis of COVID-19. The consequent increase in angiotensin II might make the AT1 predominant elderly prone to severe disease. This effect might be tempered in the AT2 predominant neonates and children leading to less severe disease.

\section{Inflammation by activation of the RAS axis}

The central role of the RAS pathway in inflammation came to light after the SARS pandemic in 2002. An elevated ACE/ACE2 activity ratio after the downregulation of ACE2 increases the available angiotensin II. AT1 receptor pathway dominates over the AT2 pathway due to its wider distribution and higher expression. ${ }^{17}$ Activation of AT1 produces different effects in the tissue-localized RAS and the RAS localized to the immune system.

The tissue effect of RAS dysregulation is multipronged in the pathogenesis of COVID-19. Activation of the AT1 receptor mediates inflammation by transcription of $\mathrm{NF}-x \mathrm{~B}$ and the production of pro-inflammatory cytokines like IL-6. ${ }^{18}$ The interaction between the S-protein of the virus and the ACE2 receptor increases CCL2 production by a separate ERK1/2 pathway. ${ }^{19}$ In mice, ACE inhibition reduced CCL2 levels and the 
recruitment of inflammatory cells. ${ }^{20}$ The latter two imply an increase in chemokine production leading to the homing of macrophages and dendritic cells. Evidence that tissue inflammation occurs due to the recruitment of inflammatory cells rather than direct T-cell activation comes from mouse models. Studies have shown that AT1 mediated tissue injury occurs despite the transplant of AT1 devoid bone marrow. ${ }^{21,22}$ Angiotensin II can lead to early neutrophil accumulation through IL-8 production as studied in human umbilical vein endothelial cells. ${ }^{23}$ ACE activity also enhances neutrophil activation and reactive oxygen species production. ${ }^{24}$ These pathways stimulate tissue-specific inflammation leading to ARDS or acute cardiac injury.

The effect of AT1 activation on the immune system is different; it inactivates the immune system. AT1 knockout T-cells in mice showed increased T-bet expression, producing more IFN- $\gamma$ and TNF- $\alpha .{ }^{25}$ AT1 knockout macrophages in mice also produced more TNF and IL-1 $\beta .{ }^{26}$ The stimulation of AT1 on T-cells and macrophages curbed their polarization.

The above research suggests that RAS activation in COVID-19 damages tissue by recruiting inflammatory cells rather than direct activation of the immune system. This might throw light on single organ dysfunction (like ARDS) in patients with well-preserved function of other organ systems. Tempering and negative regulation of T-cells, and increased neutrophil recruitment explains the poor prognosis in patients with increased neutrophil to lymphocyte ratio.

\section{Renin and prorenin in the context of COVID-19}

Renin is formed by proteolytic or non-proteolytic cleavage of prorenin. While renin production can undergo acute changes due to feedback, prorenin is constitutively secreted. Prorenin levels are elevated in hypertensives (with elevated renin) and diabetics (out of proportion to renin). Renin and prorenin ligate with the prorenin receptor that is present in the heart, kidney, brain, eyes, and on human macrophages and T-cells. ${ }^{27}$

PRR stimulation by renin/prorenin yields a pro-inflammatory response independent of the angiotensin II pathway. They stimulate the ERK1/2 pathway to increase the production of IL- 6 and COX-2. ${ }^{28}$ The proinflammatory action of PRR activation has also been depicted in mouse models of sepsis. Mice treated with PRR blockers had a significantly higher survival rate with reduced production of IL-1 $\beta$ and TNF. ${ }^{29}$

Angiotensin II-AT1 interaction is a negative regulator of renin release as per classical teaching. The intricacies of the RAS polypeptides, however, suggests differential activation that might be trigger based. AT1 activation upregulates PRR by increasing the binding of cAMP response element-binding protein to the PRR promoter ${ }^{30}$ Renin secretion and PRR activation are also upregulated by prostaglandins (PGE2) which is a consequence of COX-2 induction. ${ }^{31}$

The above-mentioned steps might be the key to a positive feedback loop in the RAS. This loop might be the trigger for a hyper-immune response that leads to the severe manifestations of COVID-19, including a cytokine storm. The higher expression of prorenin in diabetics and hypertensives supports the increased mortality in these groups of patients.

\section{Coagulation abnormalities in COVID-19}

$71 \%$ of COVID-19 non-survivors had an ISTH DIC score greater than $5 .{ }^{32}$ Coagulopathy is a major complication of severe COVID-19 infections. Though highly speculative, with few studies showing concrete proof, the RAS and the coagulation pathways are intimately linked.

RAS is intimately linked to the bradykinin and coagulation pathway by ACE, which cleaves bradykinin into inactive metabolites. In vivo dimerization of the AT1 receptor and the bradykinin 2 (BK2) receptor is also known. This heterodimerization has been best studied in the context of pre-eclampsia where an augmented response to angiotensin II is seen. ${ }^{33}$ Conditions that cause heterodimerization have not been described in literature so far. It is, however, worth noting that angiotensin II binds to this receptor conformation to produce effects similar to bradykinin.

Bradykinin binds to endothelial cells causing vasodilatation and leakage of components of the coagulation pathway (kallikrein and Hageman factor) into the bloodstream. Of note, is the potential for kallikrein and 
Hageman factor to activate prorenin. ${ }^{34}$

Plasma renin levels positively correlate with fibrinogen, plasminogen activator inhibitor, and D-dimer levels. This correlation was not found with ACE levels. ${ }^{35}$ This further strengthens the evidence for the involvement of renin in COVID-19 pathogenesis.

\section{Drug Targets}

Therapeutically, upstream regulation of RAS using aliskiren or global RAS blockade with a combination of ACEi and ARBs have not shown many benefits. ${ }^{36}$ This has been studied in the context of chronic low-grade activation of the RAS axis. A different strategy may be required in the case of a RAS-crisis or acute RAS dysregulation. The following drug targets may be viable in the setting of severe COVID-19 infections.

1. The upsurge of Angiotensin II due to acute ACE2 downregulation in COVID-19 would cause a shift in the prorenin/renin balance. Blocking the alternative pathway of RAS activation in the form of prorenin receptor antagonists will help in curbing alternate pathways of inflammation in the dysregulated RAS situation.

2. Recombinant ACE2 administered either systemically or locally to the lungs may help replenish ACE2 and restore balance to the tipped RAS axis.

3. RAS inhibition in the form of ACE inhibitors and ARBs have shown inconsistent effects in the presentation and course of COVID-19. This is because the RAS effects of the available drugs is different. This is due to the differences in the pharmacokinetics and pharmacodynamic properties of the different drugs. In the setting of an acute RAS dysfunction, we believe that global RAS blockade may help curb the excessive inflammation due to the RAS positive feedback loop.

While the initiation of the pathogenesis occurs because of the viral replication, severe cases occur due to severe immune system dysfunction. We insist that the RAS axis plays a major role in the immune dysregulation and may be targeted in the treatment of COVID-19.

\section{Conclusion}

A dysregulated RAS axis is central to the pathogenesis of COVID-19. The rapid deterioration of patients and the existence of severe manifestations, call into question the presence of a positive feedback loop (or the suppression of negative feedback). The existence of such a loop in RAS might warrant an investigation into the peptides present higher up in the pathway such as renin and prorenin. Knowledge about the functions of these molecules and their role in disease is still in its infancy. This paper has tried to elucidate possible mechanisms for the involvement of RAS in the pathogenesis of COVID-19.

Most of the studies discussed have delved into RAS as a mediator for hypertension, cardiovascular disease, and kidney disease. We believe that their results may be extrapolated into the acute context of RAS disruption in COVID-19. The lack of severe activation with positive feedback does not exist in the context of chronic diseases like hypertension; Though the existence of renal crisis in scleroderma and sympathetic crashing acute pulmonary edema might indicate otherwise. The reason for this "RAS-crisis" in the context of COVID-19 is beyond us, but we imagine is worth considering.

\section{Acknowledgement}

The figure has been created using BioRender.com.

\section{Conflict of Interest}

None

\section{References}

1. Hu Y, Sun J, Dai Z, et al. Prevalence and severity of corona virus disease 2019 (COVID-19): A systematic review and meta-analysis. J Clin Virol Off Publ Pan Am Soc Clin Virol . 2020;127:104371. doi:10.1016/j.jcv.2020.104371 
2. Hoffmann M, Kleine-Weber H, Schroeder S, et al. SARS-CoV-2 Cell Entry Depends on ACE2 and TMPRSS2 and Is Blocked by a Clinically Proven Protease Inhibitor. Cell . 2020;181(2):271-280.e8. doi:10.1016/j.cell.2020.02.052

3. Hanff TC, Harhay MO, Brown TS, Cohen JB, Mohareb AM. Is There an Association Between COVID-19 Mortality and the Renin-Angiotensin System - a Call for Epidemiologic Investigations. Clin Infect Dis . doi:10.1093/cid/ciaa329

4. Millet JK, Whittaker GR. Physiological and molecular triggers for SARS-CoV membrane fusion and entry into host cells. Virology . 2018;517:3-8. doi:10.1016/j.virol.2017.12.015

5. Peng L, Liu J, Xu W, et al. SARS-CoV-2 can be detected in urine, blood, anal swabs and oropharyngeal swabs specimens. J Med Virol . n/a(n/a). doi:10.1002/jmv.25936

6. Rockx B, Kuiken T, Herfst S, et al. Comparative pathogenesis of COVID-19, MERS, and SARS in a nonhuman primate model. Science. Published online April 17, 2020. doi:10.1126/science.abb7314

7. Glowacka I, Bertram S, Herzog P, et al. Differential Downregulation of ACE2 by the Spike Proteins of Severe Acute Respiratory Syndrome Coronavirus and Human Coronavirus NL63. J Virol . 2010;84(2):11981205. doi:10.1128/JVI.01248-09

8. Capettini LSA, Montecucco F, Mach F, Stergiopulos N, Silva RASS and RF da. Role of Renin-Angiotensin System in Inflammation, Immunity and Aging. Current Pharmaceutical Design. Published February 29, 2012. Accessed April 30, 2020. http://www.eurekaselect.com/76402/article

9. Chappell MC. Biochemical evaluation of the renin-angiotensin system: the good, bad, and absolute? Am J Physiol Heart Circ Physiol . 2016;310(2):H137-152. doi:10.1152/ajpheart.00618.2015

10. Nguyen G, Delarue F, Burcklé C, Bouzhir L, Giller T, Sraer J-D. Pivotal role of the renin/prorenin receptor in angiotensin II production and cellular responses to renin. J Clin Invest . 2002;109(11):1417-1427. doi:10.1172/JCI14276

11. Navar LG, Kobori H, Prieto MC, Gonzalez-Villalobos RA. Intratubular Renin-Angiotensin System in Hypertension. Hypertension . 2011;57(3):355-362. doi:10.1161/HYPERTENSIONAHA.110.163519

12. Ichihara A, Yatabe MS. The (pro)renin receptor in health and disease. Nat Rev Nephrol . 2019;15(11):693712. doi:10.1038/s41581-019-0160-5

13. Ozono R, Wang ZQ, Moore AF, Inagami T, Siragy HM, Carey RM. Expression of the subtype 2 angiotensin (AT2) receptor protein in rat kidney. Hypertens Dallas Tex 1979 . 1997;30(5):1238-1246. doi:10.1161/01.hyp.30.5.1238

14. Wang M, Takagi G, Asai K, et al. Aging increases aortic MMP-2 activity and angiotensin II in nonhuman primates. Hypertens Dallas Tex 1979 . 2003;41(6):1308-1316. doi:10.1161/01.HYP.0000073843.56046.45

15. Chen K, Bi J, Su Y, Chappell MC, Rose JC. Sex-Specific Changes in Renal Angiotensin-Converting Enzyme and Angiotensin-Converting Enzyme 2 Gene Expression and Enzyme Activity at Birth and Over the First Year of Life. Reprod Sci . 2016;23(2):200-210. doi:10.1177/1933719115597760

16. Xie X, Xudong X, Chen J, et al. Age- and gender-related difference of ACE2 expression in rat lung. Life Sci . 2006;78(19):2166-2171. doi:10.1016/j.lfs.2005.09.038

17. Abadir PM. The frail renin-angiotensin system. Clin Geriatr Med . 2011;27(1):53-65. doi:10.1016/j.cger.2010.08.004

18. Han Y, Runge MS, Brasier AR. Angiotensin II induces interleukin-6 transcription in vascular smooth muscle cells through pleiotropic activation of nuclear factor-kappa B transcription factors. Circ Res . 1999;84(6):695-703. doi:10.1161/01.res.84.6.695 
19. Chen I-Y, Chang SC, Wu H-Y, et al. Upregulation of the Chemokine (C-C Motif) Ligand 2 via a Severe Acute Respiratory Syndrome Coronavirus Spike-ACE2 Signaling Pathway. J Virol . 2010;84(15):7703-7712. doi:10.1128/JVI.02560-09

20. Hernández-Presa M, Bustos C, Ortego M, et al. Angiotensin-Converting Enzyme Inhibition Prevents Arterial Nuclear Factor- $\varkappa$ B Activation, Monocyte Chemoattractant Protein-1 Expression, and Macrophage Infiltration in a Rabbit Model of Early Accelerated Atherosclerosis.Circulation . 1997;95(6):1532-1541. doi:10.1161/01.CIR.95.6.1532

21. Hisada Y, Sugaya T, Tanaka S, et al. An essential role of angiotensin II receptor type 1a in recipient kidney, not in transplanted peripheral blood leukocytes, in progressive immune-mediated renal injury. Lab Investig J Tech Methods Pathol . 2001;81(9):1243-1251. doi:10.1038/labinvest.3780338

22. Koga J, Egashira K, Matoba T, et al. Essential role of angiotensin II type 1a receptors in the host vascular wall, but not the bone marrow, in the pathogenesis of angiotensin II-induced atherosclerosis.Hypertens Res Off J Jpn Soc Hypertens . 2008;31(9):1791-1800. doi:10.1291/hypres.31.1791

23. Nabah Yafa Naim Abu, Mateo Teresa, Estellés Rossana, et al. Angiotensin II Induces Neutrophil Accumulation In Vivo Through Generation and Release of CXC Chemokines. Circulation . 2004;110(23):3581-3586. doi:10.1161/01.CIR.0000148824.93600.F3

24. Khan Z, Shen XZ, Bernstein EA, et al. Angiotensin-converting enzyme enhances the oxidative response and bactericidal activity of neutrophils. Blood . 2017;130(3):328-339. doi:10.1182/blood-2016-11-752006

25. Zhang J, Patel MB, Song Y-S, et al. A novel role for type 1 angiotensin receptors on $\mathrm{T}$ lymphocytes to limit target organ damage in hypertension. Circ Res . 2012;110(12):1604-1617. doi:10.1161/CIRCRESAHA.111.261768

26. Ma L-J, Corsa BA, Zhou J, et al. Angiotensin type 1 receptor modulates macrophage polarization and renal injury in obesity. Am J Physiol - Ren Physiol . 2011;300(5):F1203-F1213. doi:10.1152/ajprenal.00468.2010

27. Danser AHJ, Deinum J. Renin, Prorenin and the Putative (Pro)renin Receptor. Hypertension . 2005;46(5):1069-1076. doi:10.1161/01.HYP.0000186329.92187.2e

28. Narumi K, Hirose T, Sato E, et al. A functional (pro)renin receptor is expressed in human lymphocytes and monocytes. Am J Physiol-Ren Physiol . 2014;308(5):F487-F499. doi:10.1152/ajprenal.00206.2014

29. Hirano Y, Takeuchi H, Suda K, et al. (Pro)renin receptor blocker improves survival of rats with sepsis. J Surg Res . 2014;186(1):269-277. doi:10.1016/j.jss.2013.08.004

30. Gonzalez AA, Liu L, Lara LS, et al. PKC- $\alpha$-dependent augmentation of cAMP and CREB phosphorylation mediates the angiotensin II stimulation of renin in the collecting duct. Am J Physiol - Ren Physiol . 2015;309(10):F880-F888. doi:10.1152/ajprenal.00155.2015

31. Kurtz A. Control of Renin Synthesis and Secretion. Am J Hypertens . 2012;25(8):839-847. doi:10.1038/ajh.2011.246

32. Tang N, Li D, Wang X, Sun Z. Abnormal coagulation parameters are associated with poor prognosis in patients with novel coronavirus pneumonia. J Thromb Haemost . 2020;18(4):844-847. doi:10.1111/jth.14768

33. Quitterer U, AbdAlla S. Vasopressor meets vasodepressor: The AT1-B2 receptor heterodimer. Biochem Pharmacol . 2014;88(3):284-290. doi:10.1016/j.bcp.2014.01.019

34. Sealey JE, Atlas SA, Laragh JH, Silverberg M, Kaplan AP. Initiation of plasma prorenin activation by Hageman factor-dependent conversion of plasma prekallikrein to kallikrein. Proc Natl Acad Sci . 1979;76(11):5914-5918. doi:10.1073/pnas.76.11.5914 
35. Sechi LA, Novello M, Colussi G, et al. Relationship of Plasma Renin With a Prothrombotic State in Hypertension: Relevance for Organ Damage.Am J Hypertens . 2008;21(12):1347-1353. doi:10.1038/ajh.2008.293

36. Parving H-H, Brenner BM, McMurray JJV, et al. Cardiorenal End Points in a Trial of Aliskiren for Type 2 Diabetes. N Engl J Med . 2012;367(23):2204-2213. doi:10.1056/NEJMoa1208799

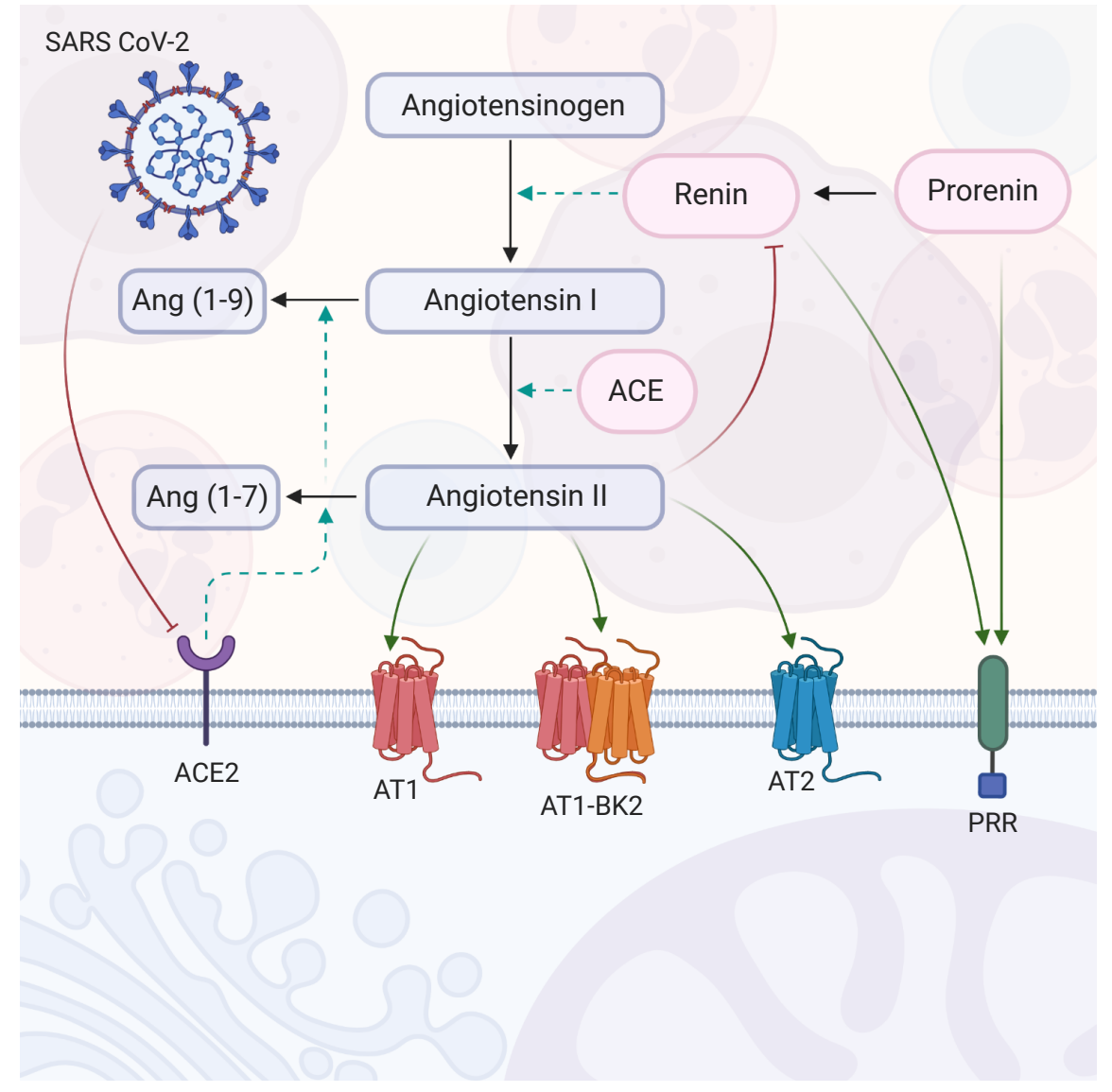

